\title{
Initial Studies on Isolation and Characterization of Antioxidant Compounds from Immature Berries of Ampelopsis grandulosa
}

\author{
Vera Fitriya Ersalena, Hiroyuki Miyazaki, Kosaku Takahashi, Koichiro Hara and Hideyuki Matsuura
}

\begin{abstract}
This initial study is aimed to isolate and characterize the antioxidant compounds from immature berries of Ampelopsis grandulosa (Vitaceae). In Japan, A. grandulosa is utilized for folk medicine because some local people in Japan believe that it has beneficial effects to human' health. However, the studies of bioactive compounds in particular antioxidant activity has not been reported for A. grandulosa. The antioxidant activities were monitored using 2,2-dyphenyl-1-perylhydrazyl (DPPH) method. The chemical structures of active compounds were determined using spectroscopic techniques such as GC-MS, ${ }^{1} \mathrm{H}$ NMR, ${ }^{13} \mathrm{C} / \mathrm{DEPT}$ NMR, COSY, $\mathrm{HMQC}$, and HMBC. A mixture of fatty acids containing palmitic acid, stealic acid, oleic acid, palmitoleic acid, trans-palmitoleic acid, and linoleic acid exhibit the radical scavenging activity. In addition, (+)-catechin and ethyl gallate have been also isolated as powerful antioxidant.
\end{abstract}

Keywords - Antioxidant compounds, DPPH, radical scavenging, Ampelopsis grandulosa.

\section{INTRODUCTION}

In the study of natural products, identify the activity of antioxidant as molecules has becoming one of the researchers' interest, since the molecules show beneficial biological activity toward us. This approach is done by several developed researches and in some cases, the antioxidant ability was evaluated using colored persistent radicals such as 2,2-dyphenyl-1-perylhydrazyl (DPPH). By definition, antioxidants are chemical compounds which is potentially capable to retard the oxidation of an oxidizable material in spite of using very low amount (commonly 1-1000 mg/L) [1].

In general, antioxidants play a pivotal role in preventing diseases driven by free radical stress. The search of natural antioxidant with the benefit of being nontoxic has been developed to a large number of studies on the potential natural antioxidants. Furthermore, debut of new antioxidants derived from natural source is awaited since the most common synthetic antioxidants such as butylated hydroxyanisole (BHA) or butylhydroxytoluene (BHT) are suspected being harmful to

Vera Fitriya Ersalena, Hokkaido University, Japan

Hideyuki Matsuura, Hokkaido University, Japan

Hiroyuki Miyazaki (Fruit Time System Company, Tokyo - Japan)

Kosaku Takahashi (Graduate School of Agriculture, Hokkaido University Japan)

Koichiro Hara (Fruit Time System Company, Tokyo - Japan) human health [2], [3]. Dietary food contains a huge variety of free radical scavenging antioxidants including flavonoids and antioxidative vitamins such as ascorbic acid and tocopherol [4]. These compounds are particularly rich in fruits, vegetables, and beverages. Numerous epidemiological studies have reported that higher intake of fresh fruits, vegetables, and tea is associated with giving lower risk of mortality from cancer and coronary of heart diseases [5], [6]. There is currently beneficial interest in natural antioxidant and their role in human health and nutrition [7]. Those antioxidant compounds are also becoming great interest for nutritionist, since there is the potential opportunity to use the food having antioxidative ingredients as functional foods [8].

One additional point should be considered is that concerning the studies among antioxidant effects on food stability [9]. The commercial development of plants as sources of antioxidants that can be used to enhance the preservation is currently becoming major interest [10].

Recently, it was reported that Ampelopsis brevipedunculata (Maxim.) Trautv. observed the potential to decrease the hepatic injury in mice [11] and was available to treat inflammatory disease. There is a closely related species with $A$. brevipedunculata, whose plant name is A. grandulosa. The immature berries of $A$. grandulosa is known as folk medicine by some people in Asia, particularly for Japanese. In this report, we present the initial studies to identify the antioxidants derived from the immature berries of $A$. grandulosa.

\section{II.EXPERIMENTAL}

\section{A. Collection of Plant Sample}

Immature dried-fruits of Ampelopsis grandulosa was collected from experimental field of Fruit Time System Company in Tokyo, Japan.

\section{B. Isolation Antioxidant Compounds}

The immature berries were immersed in ethanol extract at room temperature for three days. The ethanol extract then fractionated on silica gel column. The DPPH scavenging activity was measured according to the method described below and the active fractions were further purified on a Sephadex LH-20 column using a mixture of methanol: chloroform (1:1) as an eluate. The purified compounds were identified by GC-MS, 
${ }^{1} \mathrm{H},{ }^{13} \mathrm{C} / \mathrm{DEPT}$ NMR, COSY, HMQC, and HMBC.

\section{Measurement of Radical-scavenging Activity}

DPPH has been used by many researchers to evaluate antioxidant activity. The DPPH shows deep purple, however when the DPPH radical was scavenged by an antioxidant through donation of a hydrogen atom or an electron, the color turned from purple to yellow.
An aliquot of antioxidant solution $(200 \mu \mathrm{L})$ will be mixed with the $100 \mathrm{mM}$ Tris- $\mathrm{HCl}$ buffer $(\mathrm{pH} 7.4,800 \mu \mathrm{L})$ and add to 1 $\mathrm{mL}$ of $500 \mu \mathrm{M}$ DPPH in ethanol (final concentration of $250 \mu \mathrm{L}$ ). The mixture is shaken vigorously and leave to stand for 20 minutes at room temperature in the dark.
(A)
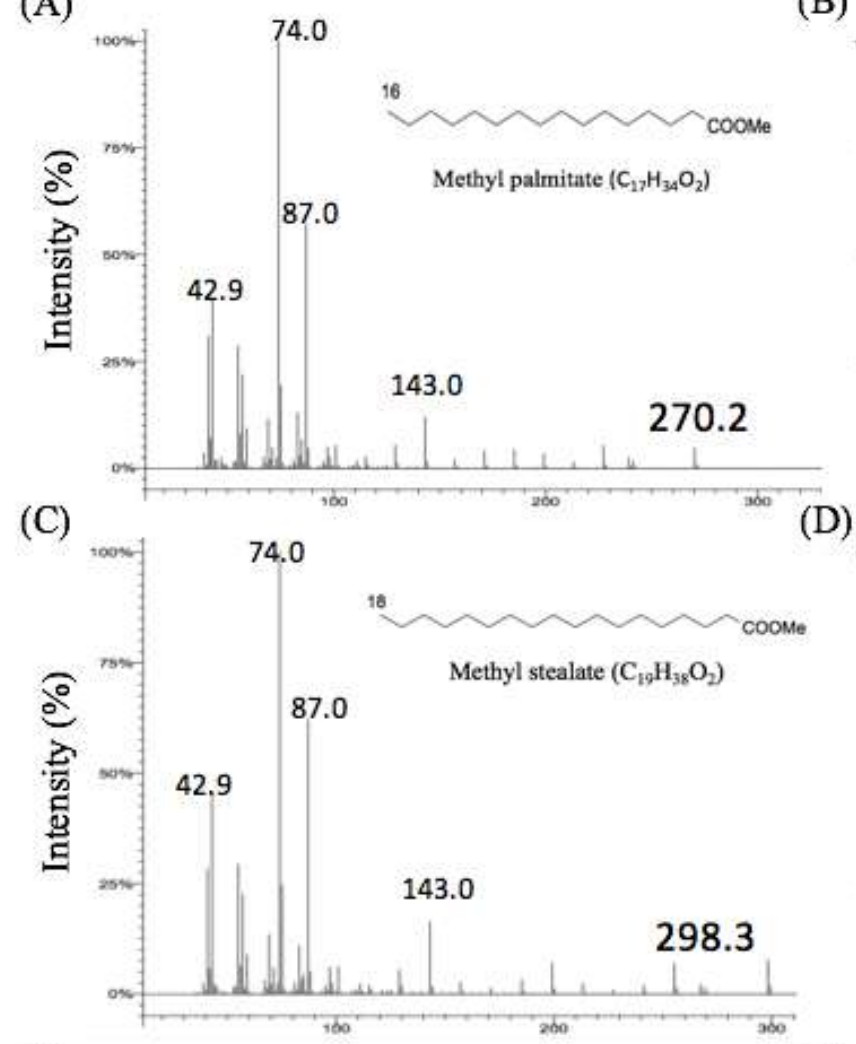

(E)

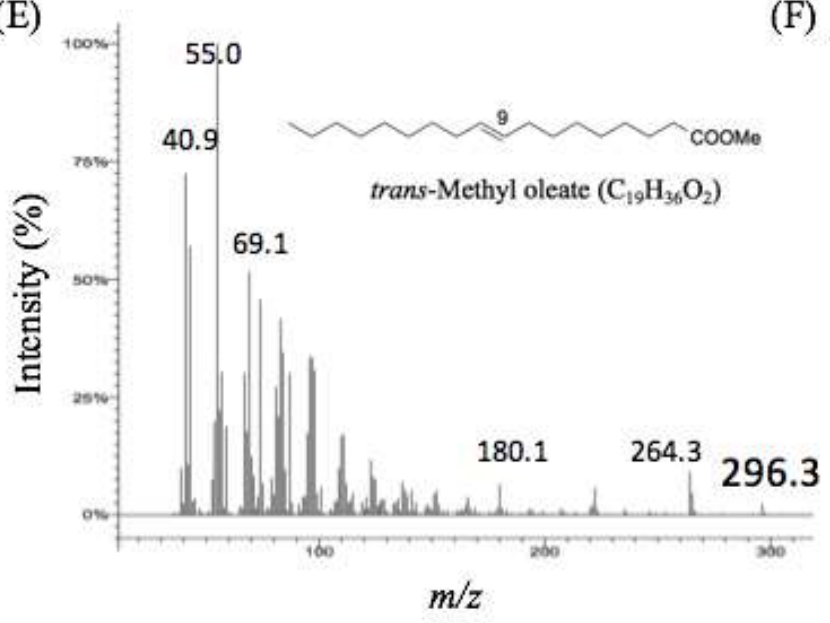

(B)

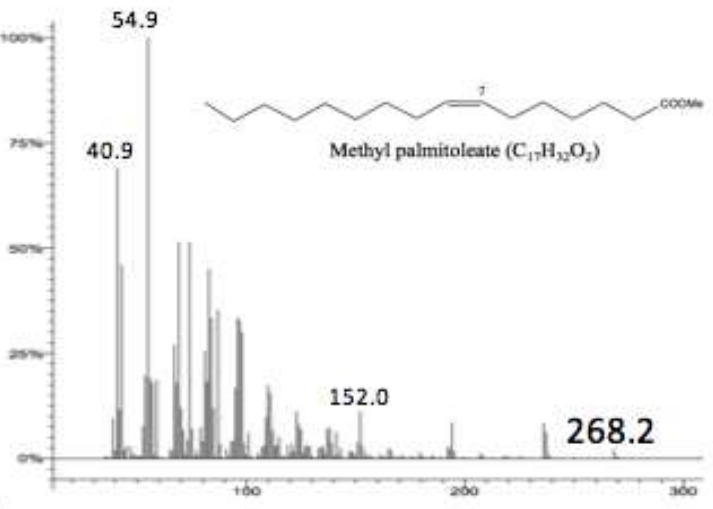

)

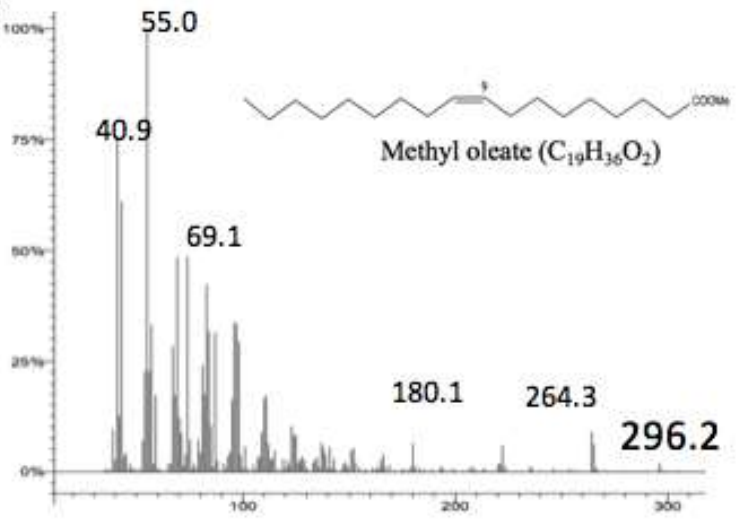

F)
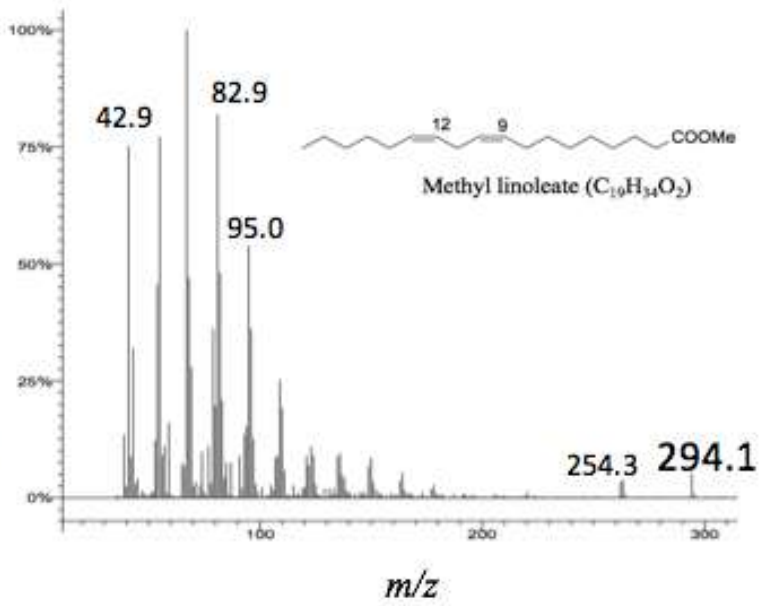

Fig. 1. Representative GC/MS Features of Fatty Acids 


\section{RESUlTS AND DisCUSSION}

In this present study, $5 \mathrm{~kg}$ of dry immature berries of $A$. grandulosa were used to isolate the compounds, and a mixture of fatty acids $(760 \mathrm{mg})$ containing palmitic acid, palmitoleic acid, stealic acid, oleic acid, trans-oleic acid, and linoleic acid are obtained by using silica gel (120 g, EtOAc-hexane, $2: 8, \mathrm{v} / \mathrm{v})$ and followed by Sephadex LH-20 column and their chemical structures were determined by GC MS analysis (Fig. 1). In Fig. 1 , the chemical structures and their GC-MS patterns are given. Since palmitoleic acid was not available as a commercial product, the compound was synthesized using 7-octyn-1-ol as a starting compound in methyl ester form according to the Scheme 1. After twenty minutes, the DPPH radical was scavenged, the color changed from purple to yellow with respectively dose of the isolated mixture $(400 ; 300 ; 200 ; 100$ $\mu \mathrm{L})$. It was indicated that fatty acids had effects on antioxidant. Although we did not determine which fatty acid(s) has activity.
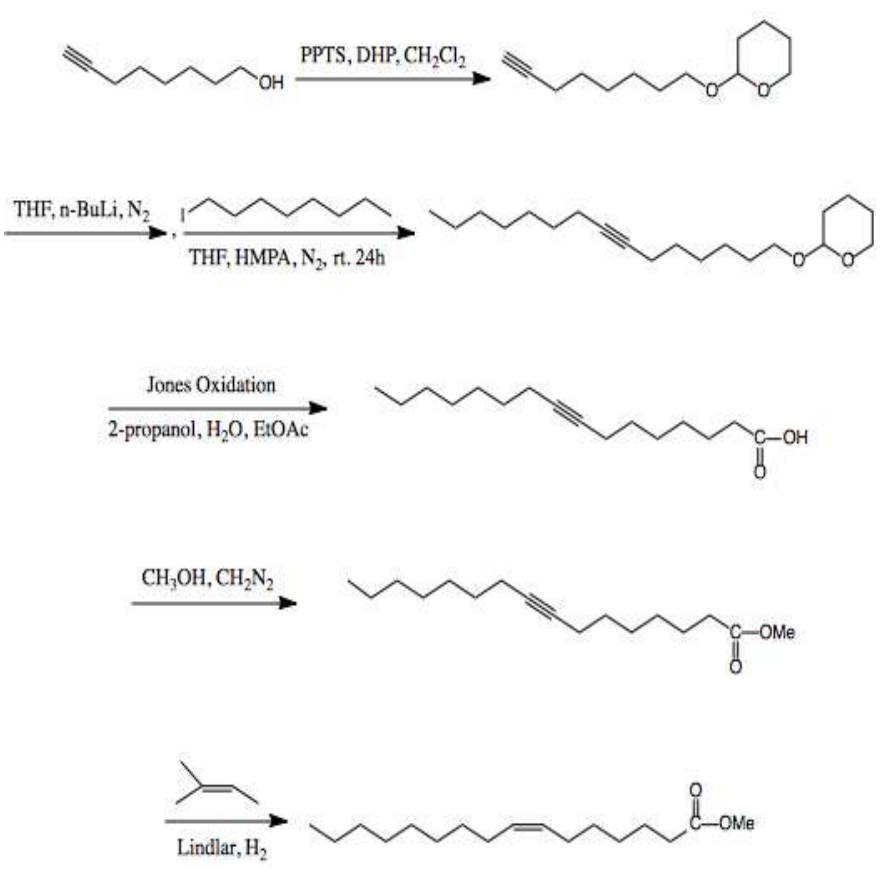

Scheme 1. Synthesis of Methyl palmitoleate

Compound $\mathbf{1}$ was isolated as yellowish oily and the molecular formula was established as $\mathrm{C}_{15} \mathrm{H}_{14} \mathrm{O}_{6}$ by HRESIMS data. The ${ }^{1} \mathrm{H}$ and ${ }^{13} \mathrm{C}$ NMR assignments obtained in methanol- $d_{4}$ were achieved primarily by the means of proton carbon correlation methods, specifically the HMBC for long range correlation and the HMQC for the direct correlation. The ${ }^{1} \mathrm{H}$ NMR data showed the presence of conjugated alkene at $\delta_{\mathrm{H}} 6.74 ; \delta_{\mathrm{H}} 6.68 ; \delta_{\mathrm{H}} 6.66$; $\delta_{\mathrm{H}} 6.63$; and $\delta_{\mathrm{H}} 6.61$ respectively (Fig. 2). Analysis of the ${ }^{13} \mathrm{C}$ NMR data and HSQC spectrum revealed 15 carbon signals in total. The carbon signal at $\delta_{\mathrm{C}} 157.0$ indicated the presence of heteroaromatics, at $\delta_{\mathrm{C}} 115$ to 120 represented the aromatic group. Ethers group indicated at $\delta_{\mathrm{C}} 69.0$ followed by alkenes group at $\delta_{\mathrm{C}} 83$, and at $\delta_{\mathrm{C}} 28.5$ referred to alkyl substituent.
According to aforementioned data, the planer chemical structure was determined to be catechin.

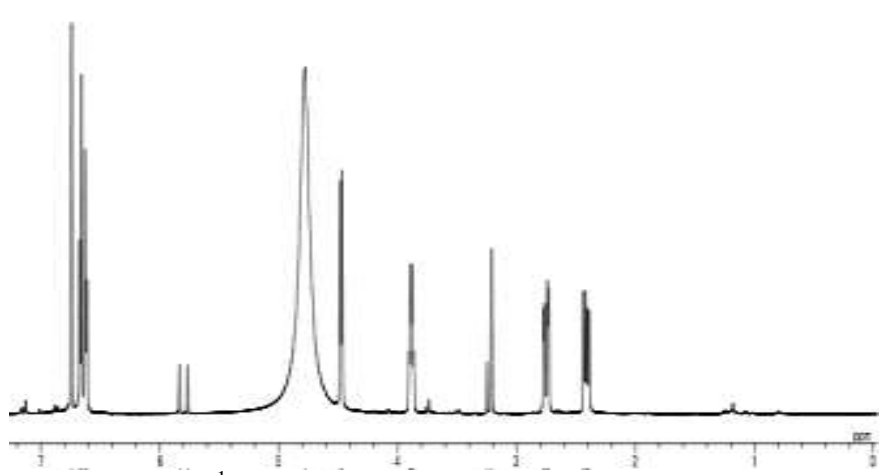

Fig. 2. ${ }^{1} \mathrm{H}$ NMR Spectrum Data of (+)-catechin (1)

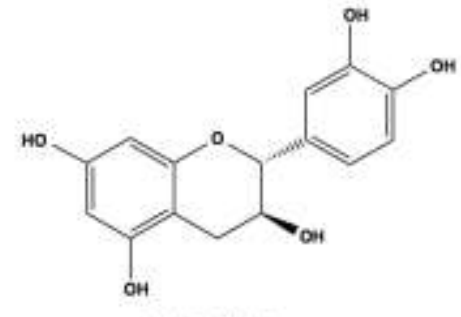

(+)-Catechin (1)

Fig. 3. Structure of (+)-catechin (1)

The absolute configuration was assigned by the measurement the specific optical rotation $\left([\alpha]_{D}\right)$, and result showed value of +11.7 which measured at $1 \mathrm{w} / \mathrm{v} \%$ concentration in $50 \%$ aqueous acetone. In terms of specific optical rotation value, it greatly depends on the solvents. In the previous studies, specific rotation of catechin was measured in three different solvents; DMF, THF, and $50 \%$ Aqueous Acetone with the values +16.9 , +3.6 , and +13.1 respectively. Then it was reported that the direction of rotation was dextrorotatory $(+)$ based on the measurement results [12]. Comparing the optical rotation values between the previous studies $\left([\alpha]_{D}=+13.1\right)$ and experimental results $\left([\alpha]_{D}=+11.7\right)$ with similar solvent $(50 \%$ aqueous acetone), there is correlation in which both indicate close values. Therefore, the structure of compound $\mathbf{1}$ was defined as (+)-catechin (Fig. 3).

(+)-Catechin (486 mg) was the major component of immature berries of A. grandulosa and seems to be responsible for antioxidant in inhibiting DPPH radical. Compare to a mixture of fatty acids, this compound is more effective as antioxidant to inhibit DPPH radical with very small dose (100; $50 ; 20 \mu \mathrm{L}$ ) respectively. This finding is in agreement with the number and arrangement of phenolic hydroxyl groups which significantly contribute as antioxidant activities against 2,2-dyphenyl-1-perylhydrazyl radical. 


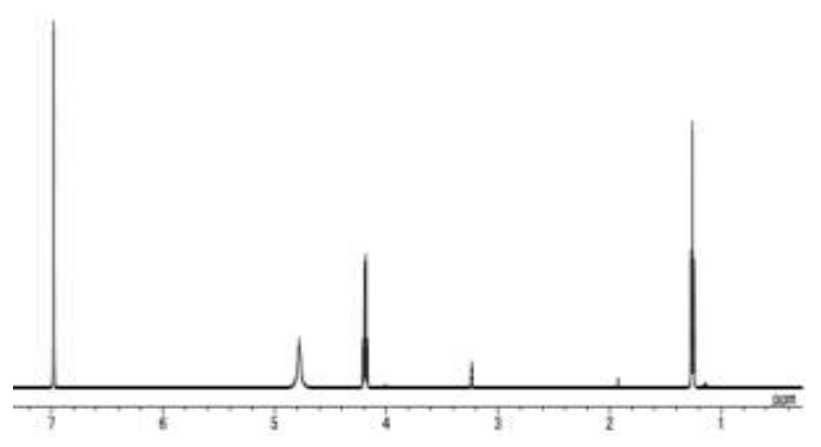

Fig. 4. ${ }^{1} \mathrm{H}$ NMR Spectrum Data of Ethyl gallate (2)

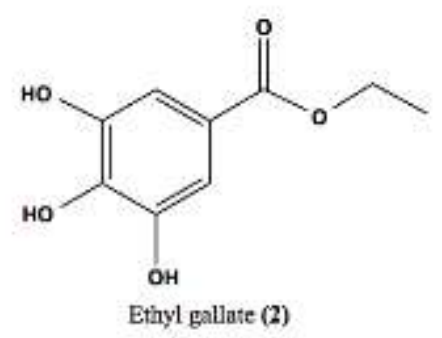

Fig. 5. Structure of Ethyl gallate (2)

Compound 2 (148 $\mathrm{mg}$ ) was also isolated as potential antioxidant, whose activities against DPPH radicals in dose of (100; 50; $25 \mu \mathrm{L})$. The ${ }^{1} \mathrm{H}$ and ${ }^{13} \mathrm{C}$ NMR measurement were obtained in methanol- $d_{4}$. The ${ }^{1} \mathrm{H}$ NMR data indicated carbon double bond at $\delta_{\mathrm{H}} 6.9$ (Fig. 4). The ${ }^{13} \mathrm{C}$ NMR data showed aromatics at $\delta_{\mathrm{C}} 121.76 ; \delta_{\mathrm{C}} 139.64 ; \delta_{\mathrm{C}} 146.42$ and acyclic hydrocarbon at $\delta_{\mathrm{C}} 14.69$. Based on the ${ }^{13} \mathrm{C}$ NMR data, it also represented the hydroxyl groups at $\delta_{\mathrm{C}}$ 61.66. Correlation between ${ }^{13} \mathrm{C}$ NMR and HMQC data defined the compound has 9 carbon signals. Hence, the compound $\mathbf{2}$ determined as ethyl gallate (Fig. 5) which has molecular formula $\mathrm{C}_{9} \mathrm{H}_{10} \mathrm{O}_{5}$.

\section{CONCLUSION}

A mixture of fatty acids which contains palmitic acid, palmitoleic acid, stealic acid, oleic acid, trans-oleic acid, and linoleic acid was identified by GC-MS analysis. The mixture identified that they have activities as antioxidant; meanwhile, we did not determine which fatty acid(s) has potential antioxidant activity. Other isolated compounds were (+)-catechin and ethyl gallate which have significant effects against DPPH in various of doses. The existence of the compounds is identified by using spectroscopic techniques. This data will be useful in further work on the characterization of antioxidant compounds from immature berries of $A$. grandulosa.

\section{ACKNOWLEDGMENT}

This study supported by Fruit Time System Company in Tokyo, Japan. Laboratory of Natural Products Chemistry, Graduate School of Agriculture, Hokkaido, Japan, and
Indonesian Endowment Fund for Education (LPDP) Scholarship, Republic of Indonesia.

\section{REFERENCES}

[1] Riccardo Amorati, Mario C. Foti, Luca Vaglimigli. Antioxidant Activity of Essential Oils. J. Agric. Food Chem. 2013, 61, 10835-10847. https://doi.org/10.1021/jf403496k

[2] Lanigan, R. S.; Yamarik, T. A. Final report on the safety assessment of BHT (1) Int. J. Toxicol. 2002, 21(Suppl. 2) 19- 94 https://doi.org/10.1080/10915810290096513

[3] EFSA Panel on Food Additives and Nutrient Sources Added to Food (ANS). Scientific Opinion on the reevaluation of butylated hydroxytoluene BHT (E 321) as a food additive. EFSA J. 2012, 10, 2588- 2630. https://doi.org/10.2903/j.efsa.2012.2588

[4] Y. Hanasaki, S. Ogawa, and S. Fukui, Free Radical Biol. Med., 16, 845-850 (1994). https://doi.org/10.1016/0891-5849(94)90202-X

[5] M. G. L. Hertog, E. J. M. Feskens, P. C. H. Hollman, M. B. Katan, and D. Kromhout, Lancet, 342, 1007-1011 (1993). https://doi.org/10.1016/0140-6736(93)92876-U

[6] M. G. L. Hertog, E. J. M. Feskens, P. C. H. Hollman, M. B. Katan, and D. Kromhout, Lancet, 342, 1007-1011 (1993). https://doi.org/10.1016/0140-6736(93)92876-U

[7] O. I. Aruoma, Food Chem. Toxic., 32, 671-683 (1994). https://doi.org/10.1016/0278-6915(94)90011-6

[8] Doriane, R., Kaouthar, K., Ullah, B., Pedro Bausero., Francesco, V., Polyunsaturated fatty acids as antioxidants. Pharmacological Research 57 (2008) 451-455

https://doi.org/10.1016/j.phrs.2008.05.002

[9] Liang, J., Tian, Y.-X., Yang, F., Zhang, J.-P. \& Skibsted, L. $\mathrm{H}$. Antioxidant synergism between carotenoids in membranes. Astaxanthin as a radical transfer bridge. Food chemistry 115, 1437- 1442, doi: 10.1016/j.foodchem.2009.01.074 (2009). https://doi.org/10.1016/j.foodchem.2009.01.074

[10] Catherine A. Rice-Evan, Nicholas J. Miller and George Paganga (1997) Antioxidant Properties of Phenolic Compounds, trends in plant science Review Vol. 2, No. 4.

[11] Yabe, N., Matsui, H., Tanaka, K., 1997. An ethanol-extract of Ampelopsis brevipedunculata (Vitaceae) berries decreases ferrous iron-stimulated hepatocyte injury in culture. Journal of Ethno-pharmacology 59, 147-159. https://doi.org/10.1016/S0378-8741(97)00121-9

[12] Hiroshi, U. Synthesis and Applications of Catechin-Aldehyde Polycondensates. J. Synth. Org. Chem., Japan. Vol.64 No.11 2006. 1192. 